\title{
Epitaxial Deposits of Metals Evaporated on Salt Substrates
}

\author{
Olive G. Engel
}

\begin{abstract}
Experimental data and theoretical considerations relating to the process of epitaxy are reviewed. The case of epitaxy resulting from vapor phase deposition of metals on rock salt is considered in detail and it is concluded that a factor other than best fit determines the orientation. A model for epitaxy in metal-rock salt pairs is advanced which involves ionization of the metal atoms on the surface. The epitaxial temperature is found to be related to the ionization potential of the metal for the ionization state to be expected. It is found that the observed orientations of metals on rock salt are those that would be expected if such an ionization process occurred, that is, if the orientation involved charged ions rather than neutral atoms.
\end{abstract}

\section{Introduction}

The increasing importance that is being attached to thin films has been pointed out by Holland [1], ${ }^{2}$ and a number of applications have been cited by Lewis [2], who arbitrarily defines "thin" to mean of the order of 100 A. Recently the Electron Physics Section of the National Bureau of Standards has needed films that are not only thin by this criterion but that also retain the perfect orientation of a single crystal. Films of this kind were required both for the electron interferometer of Marton [3] and for experiments on the single inelastic scattering of electrons.

For the interferometer a thickness of $100 \mathrm{~A}$ is permissible, but the single-crystal structure must be perfect enough so that the diffraction pattern will consist of sharply defined spots when an electron beam is passed through the film. For the scattering experiments, films of a single chemical species were desired, and to make negligible the possibility of plural and multiple inelastic scattering, a thickness of less than $100 \mathrm{~A}$ was indicated. It appeared that single-crystal metal films of this degree of thickness would be satisfactory for both applications, and that such films might be grown successfully on a substrate that would orient the growing deposit. Knowledge gained from an analysis of the experimental data on the formation of such films, along with new additions to the theory, are presented in this review of the process of epitaxy.

\section{Analysis of Existing Data}

The term epitaxy has been coined by Royer [4] to designate the oriented overgrowth of one crystalline substance on another. Although Royer's work was concerned entirely with growing a salt from its aqueous solution onto a single crystal of a different salt, epitaxy may also result when the deposit material is condensed from the vapor phase onto the substrate, when the deposit material is electrodeposited onto the substrate, or when the deposit material

1 Work sponsored by the Office of Naval Research.

2 Figures in brackets indicate the literature references at the end of this paper. is produced as a product of a chemical reaction that takes place on the surface of the substrate.

Results of past experimental work have indicated two general conditions that are necessary for the formation of oriented single-crystal metal films. These are (1) a certain amount of fit between the lattice of the substrate and that of the deposit material, and (2) a characteristic substrate temperature (epitaxial temperature) associated with every metalsubstrate pair below which perfect orientation is not obtained.

For use in the interferometer and in the scattering experiments, the film must be removed from the substrate. Rock salt, which is easily obtained in single-crystal form, and which can be removed from the metal films by solution in water, is a satisfactory substrate from this standpoint. By using substrate temperatures that are tabulated in the literature for about six metal-rock salt pairs, very thin films of a number of these metals have been condensed from the vapor phase by J. A. Suddeth and T. F. McCraw of the Electron Physics Section of the National Bureau of Standards. Not all of these films have desirable properties. The gold films are very nicely oriented if the rock salt cleavage face is maintained at $400^{\circ} \mathrm{C}$ during the deposition of the metal. They are transparent; blue light is transmitted. They have, however, the undesirable property that they collapse under their own weight at this extraordinary thickness because of the low interatomic forces of attraction between the gold atoms. Figure 1 is the electron diffraction pattern of one of the few gold films of this order of thickness that were successfully mounted by Suddeth and McCraw.

The electron diffraction patterns of silver films show that these films are well oriented if the cleavage face of the rock salt is maintained at $150^{\circ} \mathrm{C}$ during the deposition of the metal. Silver films of this order of thickness are sturdy but are full of small holes. See, for example, the electron micrograph shown in figure 2, where the angular structure of the holes (angles of 45 and 90 degrees) clearly indicates the cubic lattice type for silver. Johnson [5] has followed the structural changes of thin oriented silver films that were condensed in a vacuum on rock-salt cleavage faces as a function of temperature. For films of 1000 to $2000 \mathrm{~A}$ he found that rectangular 


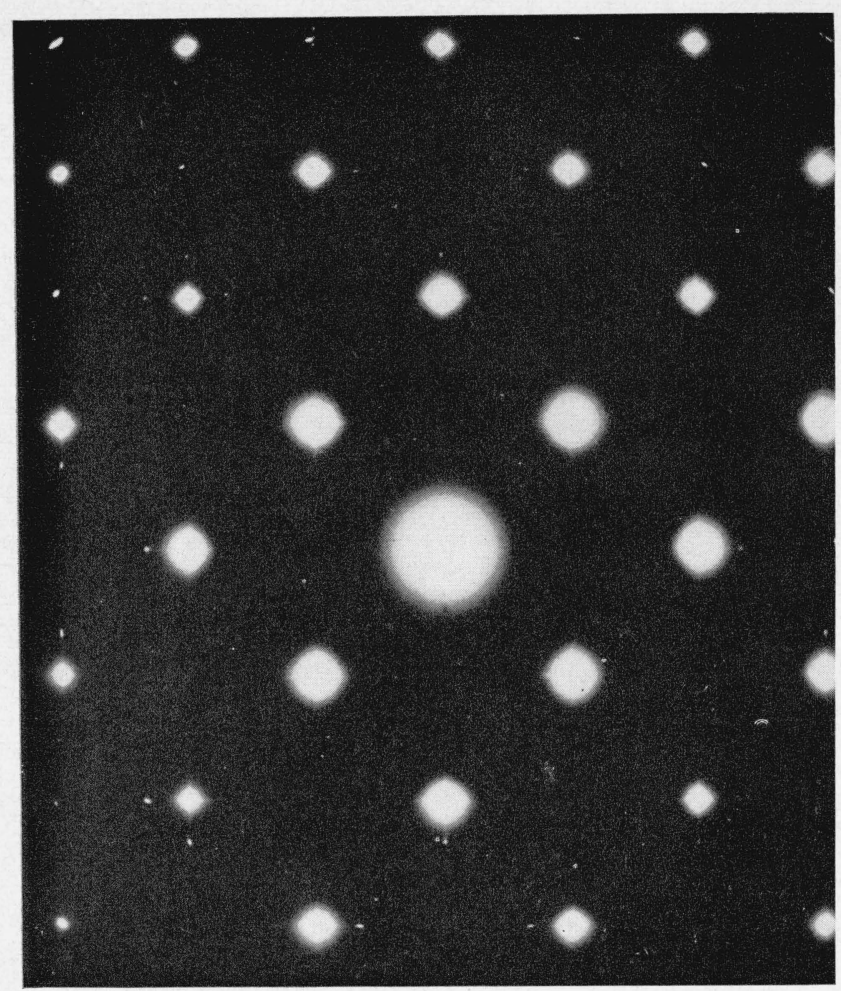

FIGURE 1. Electron diffraction pattern of a thin gold film evaporated onto the cleavage face of rock salt.

holes appeared in the films. Their edges were alined with the [110] direction of the rock salt. He attributed the effects to the readjustment of the oriented surface under the influence of surface-tension forces.

To extend experimental investigation to a variety of metals for which data are not available in the literature, it became desirable to be able to predict reasonably well not only the best substrate for maximum orientation but also the characteristic epitaxial temperature to be expected for the pair. Generalizations comparable to the rules of Royer [4] for epitaxial deposits from solution do not exist in the case of condensation of metals from their vapors. The following approach to the problem is an analysis of existing data to find a basis on which such predic. tions can be made.

\subsection{Criterion of Best Fit}

In regard to the first condition, it appears that in the case of metal films deposited from the vapor in a vacuum too much importance has been given to the degree of lattice fit between the deposit and substrate. This overemphasis of lattice fit seems to have grown out of the extensive work of Royer [4], Sloat and Menzies [6], and others on the epitaxy of salts deposited on various substrates from solution under which condition the degree of fit was apparently rather critical and the dielectric properties of the solvent played a role. It was further entrenched

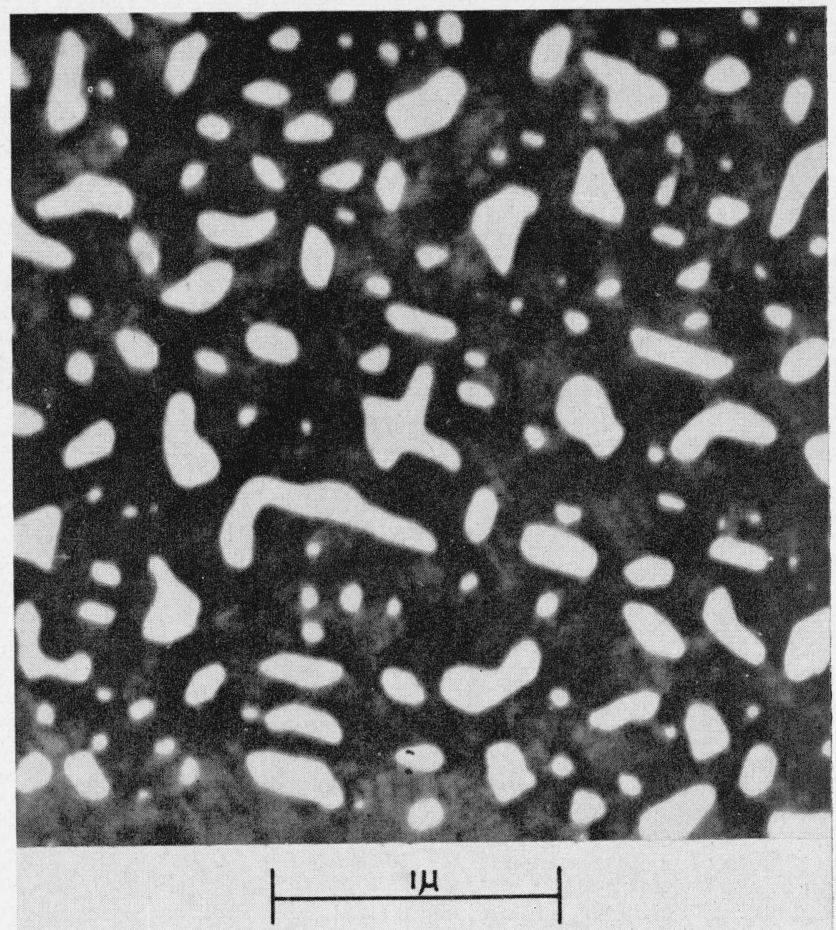

FIGURE 2. Electron micrograph of a thin silver film evaporated onto the cleavage face of rock salt.

by the mathematical treatment of Frank and van der Merwe [7, 8]. Recently, however, Schulz [9] has concluded, even for deposition from aqueous solution, that close atomic matching at the interface is not required for an oriented overgrowth. $\mathrm{He}$ obtained oriented films by deposition from the vapor phase. He then dissolved these films by condensing water vapor on them. Finally, he regenerated the oriented film by evaporation of the water. In evaluating his conclusion one must bear in mind that in the method that Schulz used the ions from an oriented deposit were just barely brought into solution by condensation of water vapor onto the film without a mechanical action so that the ions of the first oriented monolayer on being brought into solution probably never strayed far from the site on the substrate that they had occupied. In this way the oriented nucleus in solution continued to exist. Pashley [10] has oriented silver chloride on mica from the vapor phase with a misfit of -24 percent in spite of the fact that Royer [4] found the misfit limit of rock-salt lattice-type salts on mica from solution to be about 15 percent. On the basis of this evidence Pashlev has suggested that the method of growth of oriented deposits is an important factor to be taken into account.

While epitaxy that results from crystallizing the deposit material from solution may take place under fairly critical conditions of fit, results of experimental work on the deposition of metal films from their 


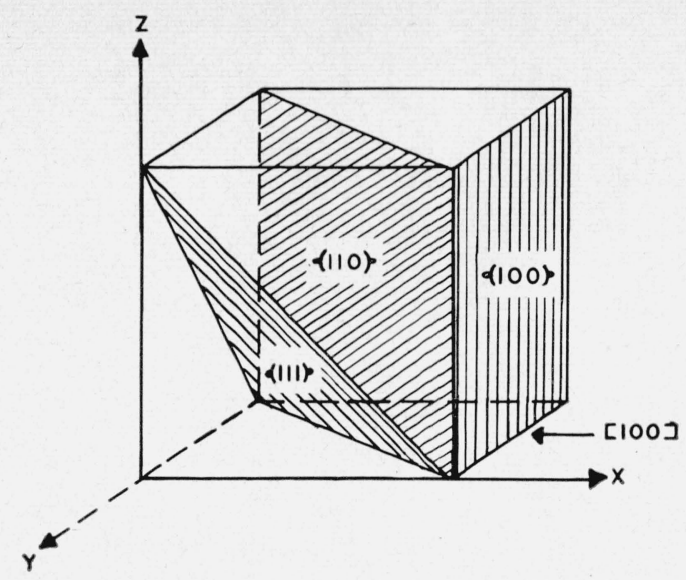

Figure 3. The $\{100\},\{110\}$, and $\{111\}$ faces of a cubic crystal structure.

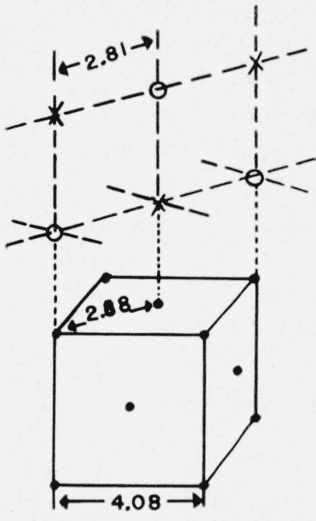

$2.5 \%$ MISFIT (NOT FOUND)

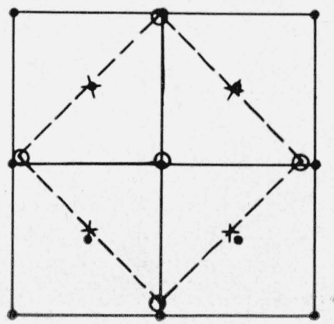

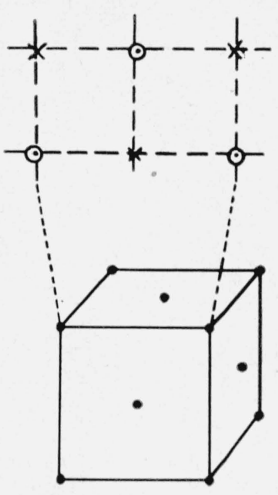

$27.5 \%$ MISFIT (FOUND)

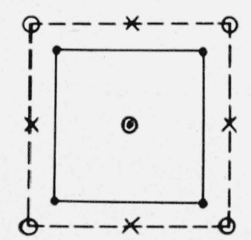

Figure 4. Silver on the $\{100\}$ face of rock salt at $150^{\circ} \mathrm{C}$.

, Silver; $\mathrm{X}$, sodium ion; $\bigcirc$, chloride ion

vapors have shown that there is a wide tolerance in fit in many cases in which epitaxy has been found, and that the depositing vapor often chooses to condense in an orientation with respect to the substrate that from the standpoint of fit would be undesirable. To illustrate the point the orientation of silver deposited on the $\{100\},\{110\}$, and $\{111\}$ faces of rock salt will be reviewed (see fig. 3).

Brück [11] in depositing silver from the vapor in a vacuum onto the cube face of rock salt at the characteristic epitaxial temperature for perfect orientation on that face found that the silver single crystal

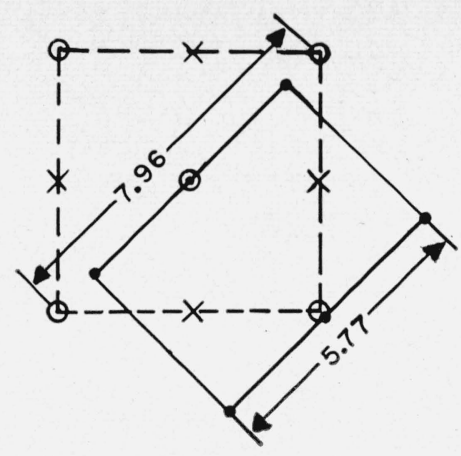

FOUND

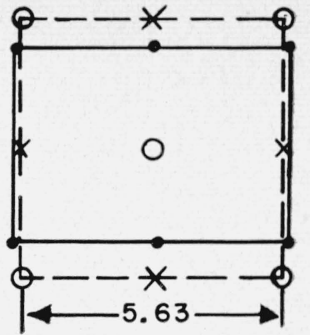

NOT FOUND
Figure 5. Silver on the $\{100\}$ face of rock salt at $450^{\circ} \mathrm{C}$.

-, Silver; $X$, sodium ion; $O$, chloride ion

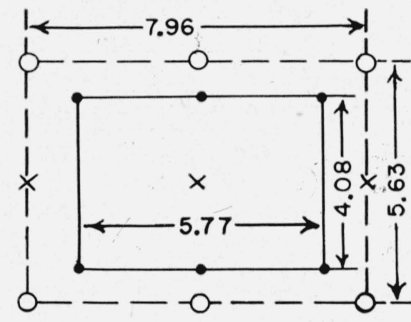

$27.5 \%$ MISFIT HORIZONTAL $27.5 \%$ MISFIT VERTICAL FOUND

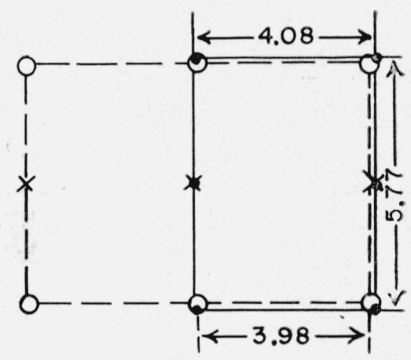

$2.5 \%$ MISFIT HORIZONTAL $2.5 \%$ MISFIT VERTICAL NOT FOUND
Figure 6. Silver on the $\{110\}$ face of rock salt

-, Silver; $\bigcirc$, chloride ion; $X$, sodium ion

grows with its $\{100\}$ face resting on the substrate and lying parallel to the cube edge of the rock salt with 27.5-percent misfit in spite of the fact that it would have a better fit (2.5-percent misfit) if it grew turned at 45 degrees to the sodium chloride cell (see fig. 4). Gharpurey [12] deposited silver on the cleavage $\{100\}$ face of rock salt at an elevated temperature $\left(450^{\circ} \mathrm{C}\right)$ and found that at this temperature the silver deposits with its $\{110\}$ face resting on the $\{100\}$ face of the rock salt. It was inclined at 45 degrees to the rock-salt cube with misfits of 2.5 percent and 27.5 percent along the two directions of the rectangular parallelogram of the $\{110\}$ face of the silver cube. From figure 5 one sees that an orientation parallel to the rock salt cube would have given the same percent misfits. It is, however, not reported.

Brú and Gharpurey [13, 14] found that when silver is deposited from its vapor onto a $\{110\}$ face of rock salt, the $\{110\}$ face of the silver rests on and is parallel to the $\{110\}$ face of rock salt. The misfit is 27.5 percent in each direction considering the chlorine lattice. If the silver were rotated through 90 degrees, the fit would be nearly perfect as is clear from figure 6 , but this orientation is not reported. Similarly, on depositing silver from the vapor onto the $\{111\}$ face of rock salt, they found that the $\{111\}$ 
face of silver was resting on the $\{111\}$ face of sodium chloride (see fig. 7 ). The misfit on the $\{111\}$ plane is 27.5 percent for the silver lattice.

Brück [11], who investigated the orientation of nine different metals on the cleavage face of rock salt, observed that whereas the cubic face-centered metals grew with their $\{100\}$ face resting on the $\{100\}$ face of the rock salt and oriented in the same way as the rock salt (i. e., with their cube edge parallel to the rock-salt cube), the cubic bodycentered metals grew on the contact surface turned at 45 degrees to the rock-salt cube. In the case of the cubic body-centered metals, then, the orientation giving best fit is observed.

From this evidence it appears that best fit is not the only criterion that determines whether or not epitaxy will occur in metals deposited from their vapors, or what orientation will be observed if it does occur. There appears to be some other factor involved that is more important than fit. An interpretation of the orientations that have been observed will be discussed at length in section 5, and the experimental observations reviewed here will be reexamined in the light of that discussion.

Brück found experimentally (by trial and error) the epitaxial temperature to produce single-crystal films of $\mathrm{Cu}, \mathrm{Pd}, \mathrm{Au}, \mathrm{Ag}, \mathrm{Ni}$, and $\mathrm{Al}$ on the $\{100\}$ face of rock salt. He tabulated these temperatures along with the lattice constant for each of the metals as is reproduced in table 1 without advancing an explanation for the differences in temperature. The most marked of these differences is that between silver and gold for which the lattice constants are nearly identical. Each of the metals listed has the cubic face-centered lattice. Three metals, Fe, Co, and $\mathrm{Cr}$, for which Brück was unable to reach the epitaxial temperature due to the fact that the rocksalt substrate sublimes at $540^{\circ} \mathrm{C}$ are not included in the table.

TABLE 1

\begin{tabular}{|c|c|c|}
\hline $\begin{array}{c}\text { Deposit } \\
\text { metal }\end{array}$ & $\begin{array}{c}\text { Lattice } \\
\text { constant }\end{array}$ & $\begin{array}{c}\text { Epitaxial } \\
\text { temperature }\end{array}$ \\
\cline { 3 - 3 } & & \\
\hline & & ${ }^{\circ}$ \\
$\mathrm{Ni}$ & 3.52 & 370 \\
$\mathrm{Cu}$ & 3.60 & 300 \\
$\mathrm{Pd}$ & 3.86 & 250 \\
$\mathrm{Al}$ & 4.04 & 440 \\
$\mathrm{Au}$ & 4.07 & 400 \\
$\mathrm{Ag}$ & 4.08 & 150 \\
\hline
\end{tabular}

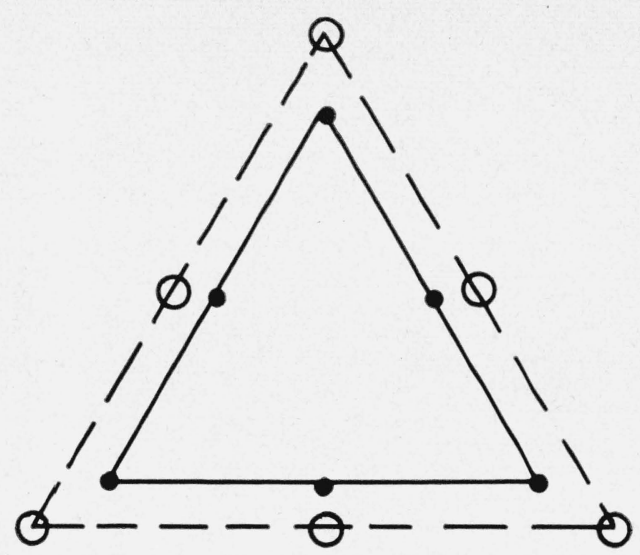

FIGURE 7. Silver on the $\{111\}$ face of rock salt. -, Silver; $\bigcirc$, chloride ion

\subsection{Epitaxial Temperature}

As has already been stated, one of the conditions for laying down an oriented deposit of a metal from the vapor phase onto a substrate is that there exists a minimum substrate temperature, the epitaxial temperature, below which perfect orientation is not observed.

Uyeda [15] has extended the investigation to a variety of substrates.

The data of Brück and those of Uyeda for the deposition of silver from tabulations given by Uyeda are listed directly in table 2 .

From this set of data it appears that the epitaxial temperature for orienting a given face parallel to the substrate (for example, the $\{100\}$ face of silver parallel to the $\{100\}$ face of galena, iron pyrite, and rock salt) is not very different for different substrates. The temperature for laying the $\{100\},\{110\}$, or the $\{111\}$ face of a given material parallel to a given substrate, however, is different. Brück observed that, in general, the most densely populated plane tends to go parallel to the substrate at a relatively low temperature; then comes the next most densely populated at a higher temperature, and so on. The most densely populated plane for cubic face-centered metals is the $\{111\}$ plane; for cubic body-centered metals, it is the $\{110\}$ plane.

The question of what determines the epitaxial temperature for a given element remains as yet

TABLE 2

\begin{tabular}{|c|c|c|c|}
\hline Substrate & $\begin{array}{c}\text { Crystal } \\
\text { type }\end{array}$ & Orientation & $\begin{array}{c}\text { Epitaxial } \\
\text { temperature }\end{array}$ \\
\hline 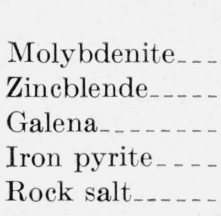 & $\begin{array}{l}\mathrm{C} 7 \\
\mathrm{~B} 3, \mathrm{~B} 4 \\
\mathrm{~B} 1 \\
\mathrm{C} 2 \\
\mathrm{~B} 1\end{array}$ & $\begin{aligned}(0001)_{\mathrm{Mos}_{2}} \|(111)_{\mathrm{Ag}} ; & {[10 \overline{10}]_{\mathrm{Mos}_{2} \|[110]_{\mathrm{Ag}}} } \\
(110)_{\mathrm{ZnS}} \|(110)_{\mathrm{Ag}} ; & {[110]_{\mathrm{ZnS}} \|[110]_{\mathrm{Ag}} } \\
(100)_{\mathrm{PbS}} \|(100)_{\mathrm{Ag}} ; & {[100]_{\mathrm{Pbs}} \|[100]_{\mathrm{Ag}} } \\
(100)_{\mathrm{FeS}_{2}} \|(100)_{\mathrm{Ag}} ; & {[100]_{\mathrm{FeS}_{2}} \|[100]_{\mathrm{Ag}} } \\
(100)_{\mathrm{NaCl}} \|(100)_{\mathrm{Ag}} ; & {[100]_{\mathrm{NaCl}} \|[100]_{\mathrm{Ag}} }\end{aligned}$ & $\begin{array}{c}{ }^{\circ} C \\
<20 \\
80 \sim 100 \\
100 \sim 150 \\
\sim 150 \\
150\end{array}$ \\
\hline
\end{tabular}


unanswered. Smollett and Blackman [16] have stated that is is hard to understand the temperature dependence of orientation in terms of critical misfit alone. Although it has been suggested that the epitaxial temperature may serve the purpose of expanding the lattice, such expansion can hardly play an important role since Eppelsheimer and Penman [17] have shown that the copper lattice constant increases only slightly over 1 percent between $18^{\circ}$ and $770^{\circ} \mathrm{C}$, which is negligible in comparison with observed misfit in epitaxy. Neuhaus [18] has associated the epitaxial temperature with the lattice energy of the deposit and has concluded that the oriented deposits are not the result of adsorption (partial energy exchange) but are the result of a true two-dimensional reaction (complete energy exchange).

\section{Process of Epitaxy}

Since the first experimental work on oriented deposits was done, attempts have been made to explain the observed orientations. At the end of a very extensive experimental investigation, Royer [4] summarized his observations in three empirical rules. These were (a) that within a certain tolerance the geometric distribution of lattice sites in the plane of the substrate and in that of the deposit should be identical or the distribution in one should be a simple multiple of that in the other, (b) that the deposit ions, which take positions that substrate ions would have taken had the substrate continued to grow, should have the same polarity that the substrate ions would have had, and (c) that the type of binding between elements of the deposit should be identical with that between elements of the substrate. He wrote the third rule with hesitancy since he was not certain that the evidence that was available really proved it. Since then, Sloat and Menzies [6] found orientation of ammonium bromide and of cesium chloride deposited from aqueous solution onto a single crystal of silver. They interpreted the observed orientation by assigning positive charges of not necessarily effectively unit value to the atoms of the silver crystal. They assumed that these charges alined the negative ions that form lattice planes in the salts. Johnson [19] found epitaxy between sodium chloride deposited from solution onto oriented films of silver, which had the $\{100\}$ and the $\{111\}$ planes parallel to the surface, and on polycrystalline silver sheet. He concluded that Royer's third rule should be modified or abandoned.

One of the earliest of the theoretical treatments of epitaxy was that of Dixit [20]. He considers that the first layer of adsorbed atoms in random lateral motion on an unattractive (amorphous) solid surface may be represented as a two-dimensional gas obeying essentially the ideal gas equation

$$
\pi A=R T \text {. }
$$

Here $\pi$ is the two-dimensional pressure, $A$ is the surface area per adsorbed molecule, $R$ is the gas constant, and $T$ is the temperature of the surface.
The two-dimensional pressure $\pi$ is given by

$$
\pi=\gamma_{0}-\gamma,
$$

where $\gamma_{0}$ is a constant and $\gamma$ is the surface tension. By substituting the value of $\gamma$ given by the RamsayShields equation, and by substituting successively the area per molecule in the $\{100\},\{110\}$, and $\{111\}$ plane, he succeeded in showing that the temperature at which any of these orientations appear is directly proportional to the area $A$ in this plane. By means of additional assumptions, he was able to calculate appearance temperatures for these planes that compared very favorably with those observed. Although his treatment applies to an unattractive substrate, the difference in the appearance temperatures of these planes should be the same on any substrate if the assumption of a two-dimensional gas can be applied to the diffusion process on the substrate surface.

Brück was aware of Dixit's work at the time that he discussed his own experimental results, but he regarded the treatment as inadequate although it was in line with his own observation that the densest plane of the deposit tends to go parallel to the substrate first. He noticed the seeming preference for misfit in the orientations of nine metals on the cleavage face of rock salt that he observed experimentally, and attempted to explain the results by saying that the orientation appeared to be such as to make the sum of the distances of the substrate ions of one species from the deposit atoms a minimum in a cell. He says that the arrangement takes place in such a manner that for the range of an elementary cell the sum of the distances of the atoms of the uppermost layer of the substrate from those of the deposit lattice will be as small as possible. Brück's rule has been supported by Uyeda, as we shall see in a moment, but it has been criticized both by Brú and Gharpurey [14] and by Thomson and Cochrane [21].

Thomson and Cochrane point out that what would be a favorable orientation in the first cell if Brück's rule were obeyed would be an unfavorable one later on. This is, of course, true, and in this respect Brück's rule is inadequate although it points in the right direction. If Brück's rule is extended, however, it can be used to define the most favorable orientation in terms of attractive bonding energy to the substrate for the largest area of monolayer deposit lattice that is capable of forming over the substrate in question. This will be discussed in more detail in section 5. Brú and Gharpurey dismiss Brück's explanation as unconvincing in that among ions of the same polarity the distances have rather to be large than small [13]. However, it does not appear to be necessary to minimize a distance-sum over ions of the same polarity. It is, for example, not necessary to conceive of the $\{111\}$ face of rock salt as being made up of sodium ions. To be absolutely realistic, the best $\{111\}$ face that one could cut experimentally would undoubtedly house both ionic species. It appears that the comment of Brú and Gharpurey must have been directed 
toward the fact that Brück, in considering the cleavage face of rock salt, imagined the sodium ions deleted from the lattice, so that a simple face-centered cubic structure of chloride ions remained. It was in a cell of this modified lattice that his rule applied. An extension of Brück's rule to include antibonding as well as bonding energy will be discussed at length in section 5 .

Thomson [22], and Thomson and Cochrane [21] thought that steps and cracks on the surface are often a determining factor in producing epitaxy. Wilman [23] rejects the explanation of Thomson and Cochrane. He states that rotational-slip results show that the existence of angles or edges of cleavage steps, whether these are macroscopic or only monatomic in height is quite unnecessary for epitaxial orientation to occur, but that if such steps or cracks are present, they may often determine the location or habit of the deposit crystals.

Uyeda [15] regards the cracks and steps as contributing only a disturbance to the ideal case. He regards the preferred orientation as being induced by the periodic field of surface force, and has advanced the following two assumptions in terms of which to discuss the oriented growth of crystals: (1) The configuration of atoms in a crystal bud formed on a base is almost the same as that formed in a free space, and (2) the interaction between the base and the crystal decides the orientation of crystals. He concludes that the orientation should be fixed while several atoms are gathering around a nucleus (compare Brück's orientation in an elementary cell) and states that this point of view supports Brück's rule of orientation and explains a few phenomena concerning orientation qualitatively. He has, however, not made clear what he means by interaction between the base and crystal. Kainuma [24] has found evidence in favor of Uyeda's conclusion.

Kubo and Miyake [25] suppose that the vaporized metal atoms, after wandering on the surface, finally arrive at the foot of a nucleus and it is from here the crystal growth commences.

The mathematical treatment of Frank and van der Merwe $[7,8]$ has already been referred to. Their theory is based on the properties of a one-dimensional dislocation model. It consists of a row of identical balls connected by identical springs having force constant $\mu$. The balls are acted on by a force that varies periodically with the position on the substrate. They let $a$ be the wavelength of the potential due to the substrate and $W / 2$ be its amplitude. The potential energy is represented by the first harmonic term (amplitude $W / 2$ ) in a Fourier series. A difference may exist between the natural spacing $b$ of the balls and the wavelength $a$ of the substrate field. From the theory for the model it turns out that when the natural spacing $b$ of the balls differs from the wavelength $a$ of the substrate field, the lowest energy state of the system is one in which there are no dislocations (small regions in which the balls would be spaced more closely or less closely than normal) up to a certain critical value of the misfit
$1 / P_{0}$, where

$$
1 / P_{0}=(b / a-1)_{\text {eritical }}=2 / \pi l_{0}
$$

and

$$
l_{0}=\left(\frac{\mu a^{2}}{2 W}\right)^{1 / 2} .
$$

By using Lennard-Jones forces, and by assuming interactions with other deposit atoms and with substrate atoms are similar, they find the value of $l_{0}$ to be about 7 and hence the critical value of the misfit to be about 9 percent. Below this critical misfit the monolayer in its lowest energy state is deformed into exact fit with the substrate. Above the critical misfit it is only slightly deformed on the average, but it contains many dislocations. Up to the larger critical misfit of 14 percent the monolayer can be deposited metastably in exact fit on the substrate at temperatures that are sufficiently low. Completely oriented overgrowth is to be expected only if the first monolayer can form over the entire surface under subcritical conditions.

Smollett and Blackman [16] have investigated the stability of a strained two-dimensional layer. They evaluated the lattice constants of a two-dimensional alkali halide layer strained to fit on an alkali halide crystal substrate. For a series of cases in which oriented overgrowth is found experimentally they found instability in the initial deposited layer if it is strained to fit the substrate. They conclude that experimentally observed orientations cannot result from an initially uniformly strained layer such as is indicated by the Frank-van der Merwe theory. They further conclude that the initial lattice must be distorted in the sense that displacements occur, but that the lattice constant of the deposit material remains more or less unchanged.

In analyzing the forces that operate in epitaxy from solution, Sloat and Menzies [6] state that the most important are the mutual attraction between the solute ions and the substrate ions, and the mutual attraction between oppositely charged solute ions. Following Lennard-Jones and Dent [26] they further analyze the first force mentioned into four parts: (1) Electrostatic attraction of the ionic charge to the valency charges of the substrate; (2) the force of attraction between substrate ions and the dipole which results from the polarization of the attracted ion by the surface field; (3) the attractive force resulting from the polarization of the surface ions by the attracted ion; and (4) the van der Waals attraction between neutral atoms. The van der Waals attraction is operative at longer distances, but at short range the electrostatic force is much greater in magnitude. They conclude that it is first the van der Waals force which is operative; then the electrostatic. They ascribe the final capture of an ion, and the fixing of the ion into the space lattice of the substrate, to the electrostatic forces.

It appears that a similar mechanism must operate in the epitaxy of metals deposited from their vapors, although Rhodin $[27,28]$ has found rather surprising agreement on the assumption of van der Waals 
forces. However, his criterion of the completion of the deposition of a monolayer was conductivity across the film. It is hard to believe that a continous monolayer of metal is ever produced because of the strong cohesive forces of the deposit. Rather it would seem that aggregates of a number of layers in depth eventually grow together. One can then hardly talk about the binding force of the first layer from a conductivity measurement.

\section{A Model for Metal-Rock Salt Pairs}

In order to establish a basis on which prediction of metal-substrate pairs for vapor-phase deposition and of their epitaxial temperatures can be made, it is necessary to know the process involved. If this process can be understood for one substrate, it can be generalized to others. With this view in mind the special case of the epitaxy of metals on a rock-salt substrate has been considered [28a] because a larger amount of experimental data exists for this substrate.

We shall start with the following assumptions: (1) Orientation must result from the interplay of forces, and (2) the preferred orientation must be the configuration of lowest energy. If we assume that the first layer of deposit-metal exists as atoms, the forces, in addition to van der Waals forces, that are involved are electrostatic forces acting between the substrate ions and the dipoles induced by the substrate ions in the neutral metal atoms. If, on the other hand, we consider that the atoms of the first layer of deposit-metal become ionized, they are electrostatic forces acting between the ions of the substrate and deposit-metal ions on the surface. When neutral metal atoms arrive on the surface of the substrate, forces of the first kind are operative. If, after arrival on the surface, the deposit-metal atoms permanently lose one or more electrons, forces of the second kind exist. A true two-dimensional reaction can then occur between the deposit-metal ions and the anions of the substrate so that at the interface the three layers - substrate salt, depositmetal salt monolayer, deposit-metal-exist. The deposit-metal salt that forms as a result of this reaction must be sufficiently mobile to diffuse on the surface possibly in the form of symmetrical units, or lattice cell fragments, such as discussed by Zener [29] until larger static aggregates are formed. It would appear that the epitaxial temperature should be related not only to the mobility of this salt, which in the form of larger aggregates is eventually firmly anchored to the substrate by true electrovalent bonds, but that the epitaxial temperature is also a function of the ionization potential of the respective metals. Thermal ionization at the expitaxial temperature is, of course, out of the question because of the vanishingly small value of the Boltzmann factor. But the combined effect of thermal energy and the availability of electron traps on the surface could be sufficient as we shall see in a moment. If one plots the first ionization potential of each of these metals against the epitaxial temperature, there is no relation between them. If, on the other hand, one plots the ionization potential of the most commonly encoun- tered ionization state, the trend is along a line (see fig. 8, and reference [32]). For these metals the only valence states used that are higher than the lowest ionization state possible for the atom in question under usual conditions are $\mathrm{Cu}$ II, $\mathrm{Au}$ III, and Ni III. We shall return shortly to a discussion of the reasonableness of assuming that these will be the most stable valence states to be expected for these metals, and we shall consider first whether or not the process that has been advanced is energetically feasible.

In considering what could constitute trapping sites for the electrons, one might at first sight fix upon the sodium ions. That this is not a logical choice, however, is evident from the fact that if rock salt is heated in sodium-metal vapor, $F$-centers are formed in preference to the alternative that the lattice should house sodium metal interstitially. The $F$-centers (Farbzentren, or color centers) are vacant chloride ion sites, which due to their effective positive charge, have trapped an electron whose orbit then extends over the six surrounding sodium metal ions. They may be regarded as a vacant negative ion site at which one of the nearest neighbor positive ions has gained the electron and become a neutral metal atom. If we write $M$ for the deposit-metal, $S$ for a vacant negative ion site, and $F$ for a color center, then we are concerned with the energy for the two-step process,

$$
\begin{array}{ll}
\text { Step (1): } & M^{0}-n e \rightarrow M^{n+} \\
\text { Step (2): } & n S+n e \rightarrow n F .
\end{array}
$$

The second step in sodium chloride involves about $2.7 \mathrm{ev}$. This is the peak of the characteristic absorption band of the $F$-centers [30]. The metal atom, let us consider it to be silver, is first adsorbed from the vapor onto the cleavage face of the rock salt. After a certain period of time, during which it is adsorbed on the surface, loss of an electron takes place. Since the silver atom is on the surface of the sodium chloride, the energy required is less than the gas phase ionization potential of silver. That is, the ionization potential is reduced because of the dielectric constant of the salt. Because a thermal rather than an optical process is involved, the static dielectric constant $K$ is in question rather than $K$ optical arising from the distortion of the electron clouds. The whole value of $K$ would be effective only if the process took place below the surface of the crystal, that is, if the adsorbed atom were completely dissolved in the rock salt. If the cleavage plane were atomically smooth, one might reasonably assume that the dielectric constant should be reduced by somewhat less than half. Actually the factor 0.44 has been used as this gives the lowest positive value for $\Delta E$ in the case of silver for which the data are known most accurately. In addition to the energy available from eq (6) there is also available the energy of placing a $\mathrm{Ag}^{+}$ion over a $\mathrm{Cl}^{-}$ion on the cleavage plane. If the cleavage plane were atomically smooth, this energy would be given by [31]

$$
E=\frac{0.066 e^{2}}{r},
$$


where $e$ is the electronic charge, and where $r$ is the $\mathrm{Ag}^{+}-\mathrm{Cl}^{-}$distance of separation. The first ionization potential of silver [32] is $7.574 \mathrm{ev}$, the dielectric constant of rock salt [30] is 5.62, and if we take the $\mathrm{Ag}^{+}-\mathrm{Cl}^{-}$distance to be the sum of the ionic radii (Goldschmidt radii are $\mathrm{Ag}^{+}=1.13 \mathrm{~A}, \mathrm{Cl}^{-}=1.81 \mathrm{~A}$ ), then

$$
\Delta E=\frac{7.574}{0.44 K}-2.7-\frac{0.066 e^{2}}{r}=0.04 \mathrm{ev} .
$$

In the case where a doubly charged ion is produced, as in $\mathrm{Pd}^{++}$, an additional energy is involved due to the polarization of the surface ions by the double positive charge. Wright [33] gives the potential when an electron is added at a positive ion site in sodium chloride as $-2.5 \mathrm{ev}$. It would appear that on the surface, although the $\mathrm{Pd}^{++}$ion has only one nearest neighbor $\mathrm{Cl}^{-}$ion, it sees all of the ions in the plane on which it is standing, so that the polarization energy is a large fraction of that which results when an electron is given to a positive ion in the interior of the salt. A reasonable guess might be 0.7 of this value or $1.75 \mathrm{ev}$. In the case of forming a doubly charged ion, two $F$-centers are involved and the energy for placing the ion on a $\mathrm{Cl}^{-}$ion in the plane is doubled. Because the second ionization potential of palladium is $19.42 \mathrm{ev}$ [34], the energy for producing the $\mathrm{Pd}^{++}$ion is given by

$\Delta E=\frac{19.42}{0.44 K}-2(2.7)-2\left(0.066 e^{2} / r\right)-0.7(2.5)=0.01$.

There is little point in estimating the energy change for a triply charged ion such as $\mathrm{Au}^{+++}$because of the uncertainty both in the value of the ionization potential and in the polarization energy.

This value of $\Delta E$, however, does not determine the equilibrium constant. It is really the thermodynamic $\Delta E_{0}^{0}$ or energy change for the reaction at the absolute zero of temperature. The equilibrium constant $K$ is given by

$$
-\ln K=\Delta F^{0} / R T \text {, }
$$

where $R$ is the gas constant, $T$ is the absolute temperature, and $\Delta F^{0}$ is the standard free-energy change.

$$
\Delta F^{0}=\Delta E_{0}^{0}+\Delta\left(\frac{F^{0}-E_{0}^{0}}{T}\right) T .
$$

For the ionization process postulated, the second term on the right for silver is

$$
\begin{aligned}
T\left[\left(\frac{F^{0}-E_{0}^{0}}{T}\right)_{\mathrm{Ag}+}+\right. & \left(\frac{F^{0}-E_{0}^{0}}{T}\right)_{\text {F-center }}- \\
& \left.\left(\frac{F^{0}-E_{0}^{0}}{T}\right)_{\mathrm{Ag}^{0}}-\left(\frac{F^{0}-E_{0}^{0}}{T}\right)_{\text {vacant-site }}\right] .
\end{aligned}
$$

This information is not available and consequently the equilibrium constant cannot be calculated.
If it is conceded that ionization of the deposit atoms does occur on the surface of the substrate, and the evaluation of the energies involved appears to indicate that such a process is feasible, then it must also be conceded that the epitaxial deposits consist of the layers: rock salt, deposit-metal salt, depositmetal, of which the deposit-metal salt layer is a monolayer. If this is indeed true, we must expect in every case in which epitaxy is observed that there is epitaxy between rock salt and the deposit-metal salt as well as epitaxy between the deposit-metal salt and the deposit-metal, and we must consider the lattice fit between the salt lattices, and that between the deposit-metal salt lattice and the deposit-metal lattice rather than the fit between the metal lattice and the rock-salt lattice as has been done so far.

Following this point of view, when the silver metal itself deposits, it does so on an oriented surface of silver ions. Here, three cell lengths of the silver chloride lattice $(16.62 \mathrm{~A})$ are closely equivalent to four cell lengths of bulk silver $(16.32 \mathrm{~A})$. The ease with which the films can be floated from the salt cakes seems to indicate that they are not bonded to the substrate cell by cell such as an additional layer of sodium chloride would be. Rather, there must literally be tunnels through which the water can flow under the film.

Some very interesting experimental work has been done recently by Johnson [35] in regard to epitaxy of salts on metal surfaces. He found epitaxy between rock salt and the following metals: $\mathrm{Cu}, \mathrm{Ag}$, $\mathrm{Au}, \mathrm{Zn}, \mathrm{Pb}, \mathrm{Sb}$, and $\mathrm{Bi}$, when he evaporated a salt solution on the metal. Two metals known to exhibit epitaxy when deposited from the vapor onto a rocksalt cleavage plane were negative to Johnson's test. These were nickel and aluminum. While one might ascribe the negative result in the case of nickel to the large misfit between the nickel lattice and the rock salt lattice, one could hardly advance this argument in the case of aluminum where the lattice constant is very close to that of silver and gold. The answer may then possibly be that it is not necessary that there should be epitaxy between rock salt and the metal itself, but that it is only necessary that there should be epitaxy between rock salt and the metal salt; and again, epitaxy between the metal salt and the metal as has just been indicated. Johnson explained his results by assuming the presence of an oriented oxide layer on the metal. Where the oxide layer was known to be amorphous, orientation did not occur. In the case of silver and gold no oxide layer was considered to be present. In this connection Johnson states that it is possible then that the oriented growth of sodium chloride on silver did involve an intermediate thin layer of silver chloride. Silver produced a much better orientation of the sodium chloride than did the gold. In regard to this, Johnson states that the difference in behavior of silver and gold must be attributed to differences in their chemical activity. Brück [11] also found that silver was a more efficient substrate material than gold as is evident from the epitaxial temperatures that he found for deposit-substrate pairs given in table 3. 
TABLE 3

\begin{tabular}{|c|c|c|}
\hline Substrate & \multicolumn{2}{|c|}{ Deposit } \\
\hline & Ag & $\mathrm{Au}$ \\
\hline $\mathrm{NaCl} \ldots \ldots . . . . . .$. & $\begin{array}{l}{ }^{\circ} \mathrm{C} \\
150\end{array}$ & $\begin{array}{l}{ }^{\circ} \mathrm{C} \\
400\end{array}$ \\
\hline $\mathrm{Ag}$ & 100 & 300 \\
\hline $\mathrm{Au} \ldots \ldots$. & 250 & .... \\
\hline
\end{tabular}

Sloat and Menzies [6] have shown that sodium chloride can orient silver chloride and Wilman [36] has shown that silver can orient silver chloride. Consequently, orientation is possible through the three layers: $\mathrm{NaCl}, \mathrm{AgCl}$, and $\mathrm{Ag}$. In the list of positive and negative cases of epitaxy given by van der Merwe [8] it is indicated that copper does not orient cuprous chloride. It remains to be shown that copper does orient cupric chloride since according to the plot of epitaxial temperature against ionization potential shown in figure 8 one is led to believe that the divalent salt forms. Only by obtaining similar data for the other metals for which the epitaxial temperature on rock salt is known, can it be shown that the valence state indicated by the epitaxial temperature is the only ionization state for which the metal salt will orient both the substrate salt and the metal deposit.

Of the metals so far considered (data of Brück [11]) the only valence states used that are higher than the lowest state possible for the elements in question under usual conditions were $\mathrm{Cu}$ II, $\mathrm{Au}$ III, and Ni III. In determining whether or not chlorides in these valence states would be stable at the epitaxial temperatures required, one must consider both the stabilizing effect of the sodium chloride and the fact that a monolayer is in question. Morris [37], for example, has found that auric chloride solution containing sodium chloride is stable to $450^{\circ} \mathrm{C}$ but auric chloride by itself decomposes at a much lower temperature. What effect stabilization due to the sodium chloride may have in determining the valence state that should be observed can only be surmised. The chemistry of thin layers, let alone monolayers, has been investigated very little. In regard to the use of the higher oxidation state for gold, it is noteworthy that aurous chloride undergoes dismutation with ease to produce auric chloride and gold.

The limitations of the graph shown in figure 8 must be clearly borne in mind. Each of the six metals on it has the cubic face-centered lattice type. The epitaxial temperature for each is for deposition of the $\{100\}$ plane parallel to the $\{100\}$ plane of rock salt. It is to be noted that for face-centered cubic lattices, the orientation with the $\{111\}$ plane parallel to the substrate should occur at a lower temperature than that for orientation of the $\{100\}$ plane parallel to the substrate. However, the orientation with the $\{111\}$ plane parallel to the substrate in many cases never appears. Brück, for example, observed that this was true for copper, palladium, nickel, and cobalt. He found that the $\{111\}$ orientation parallel to the substrate did appear

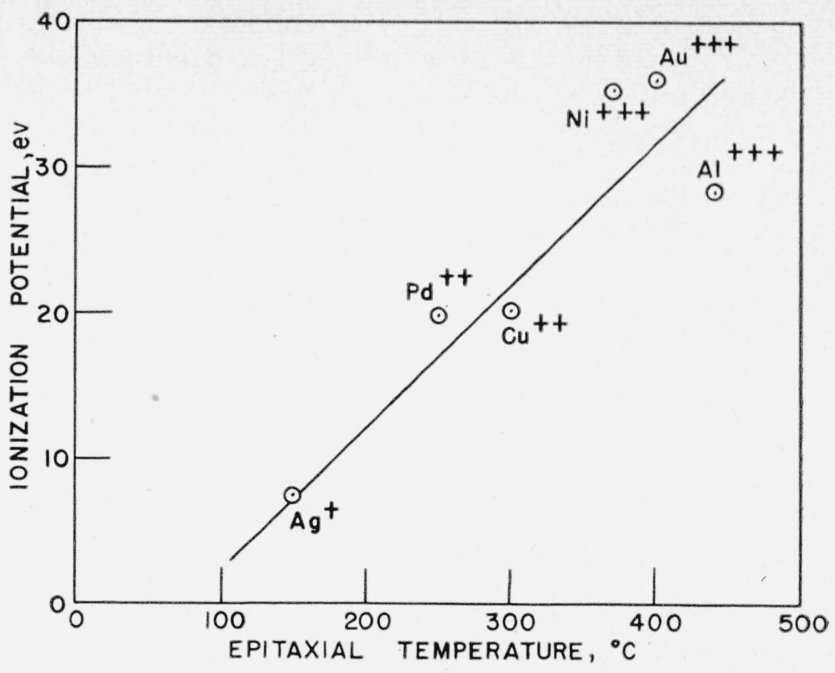

Figure 8.

for gold and aluminum and that there was some indication of its appearance for silver. The fact that it does appear in aluminum, and must be forced out by increase in temperature, may account for the poorer fit of aluminum on the graph. If the epitaxial temperature for laying down the $\{111\}$ plane of aluminum parallel to the substrate (which may be taken to be about $350^{\circ} \mathrm{C}$ since above this value the $\{100\}$ plane starts to deposit) were used for aluminum, this metal would have a much better fit on the graph. This, too, would be in line with the assumption that the metal must be ionized to be oriented at all. Gold would also be displaced to the left on this reasoning.

In the discussion of Dixit's investigation in section 3 , it was pointed out that although his treatment applies to an unattractive substrate, the differences in his appearance temperatures should be correct for any substrate if the assumption of a two-dimensional gas can be applied to the diffusion process on the substrate surface. For silver, Dixit finds the $\{111\}$ appearance temperature to be $659^{\circ} \mathrm{C}$ and that of the $\{100\}^{3}$ face $801^{\circ} \mathrm{C}$. The difference is 142 degrees. Brück reports that silver can be oriented fairly well at room temperature. At $150^{\circ} \mathrm{C}$ he obtained single crystal orientation with the $\{100\}$ face parallel to the substrate, but sometimes, in the foils nearly oriented to a single crystal, there was a light superimposition with the $\{111\}$ structure. Assuming that the first observed orientation was with the $\{111\}$ plane parallel to the rock salt, the temperature range between the two orientations is about 130 degrees. For aluminum on an unattractive substrate Dixit found for the $\{111\}$ appearance temperature $264^{\circ} \mathrm{C}$, for the $\{100\}$ appearance temperature $346^{\circ} \mathrm{C}$, and for the $\{110\}$ appearance temperature $602^{\circ} \mathrm{C}$. The difference between the appearance temperatures for the $\{100\}$ and $\{111\}$ planes is 82 degrees. In regard to aluminum Brück states that above $350^{\circ} \mathrm{C}$ the single crystal drives out gradually the $\{111\}$ structure until

${ }^{3}$ Dixit, who was considering interplanar spacings, used the notations $\{200\}$ and $\{222\}$ instead of $\{100\}$ and $\{110\}$. 
it only exists at $440^{\circ} \mathrm{C}$. The aluminum film at $440^{\circ} \mathrm{C}$ has the $\{100\}$ plane parallel to the rock-salt cleavage plane. This is a temperature range of 90 degrees, which again compares favorably with the difference in Dixit's calculated values. This agreement appears to indicate that the same assumption of a two-dimensional gas may be applied to attractive substrates.

There appear then to be two temperature dependent processes: (1) the ionization process and (2) the process by which the $\{111\},\{100\}$, and $\{110\}$ planes orient parallel to the substrate. The secord process is a complicating factor in the first. The answer to the question as to why orientation with the $\{111\}$ plane parallel to the substrate does not make its appearance for every metal is not clear. It must be energetically forbidden. The answer may be simply that the temperature required to produce ions that can be oriented at all in sufficient number is already in excess of that at which the $\{111\}$ plane would orient parallel to the substrate if ions had been available. Then the next most densely populated plane, the $\{100\}$ plane for the face-centered cubic structure, would show up.

\section{Retrospect}

In this section we return to the experimental results that have been cited for the deposition of metals from their vapors onto a rock salt substrate to find if the ionization process that has been advanced can explain the orientations which have been found. At its epitaxial temperature, Brück found that silver deposits on the cleavage face of rock salt in the way indicated in figure 4 , although, if there were a relative rotation of 45 degrees, the orientation designated as not found in figure 4 , the misfit would be very much less. It is interesting to observe from the twodimensional sketches that the misfit orientation that is actually found places a chloride ion in closest proximity to a silver atom (or silver ion, in terms of the ionization process discussed) and leaves the sodium ions with no silver close by. If the silver cube diagonal did lie along the rock-salt cube edge (the orientation involving best fit, but which is not found) a sodium ion would make a close approach to a silver atom. If the silver atom is really a silver ion, this would involve repulsion. If silver ions, rather than silver atoms, are involved, the orientation observed is in accord with Royer's second rule, which says essentially that you will not get epitaxy if ions of the wrong polarity have to be brought together. This rule is for the orientation of charged ions depositing out of solution. The fact that it indicates as impossible the orientation that is actually not found, but which one would expect to find on the basis of best fit, lends support to the idea that charged ions are involved. The orientation that is found appears to try to establish as closely as possible a monolayer lattice of silver chloride using the anions of the substrate. The lattice constant of silver chloride $(a=5.545 \mathrm{~A})$ is nearly the same as the lattice constant of sodium chloride $(a=5.628 \mathrm{~A})$. A close approach to this hypothesis was made by Drabble [38] who concluded that the epitaxy is such that the nearest possible coordination fit between the silver chloride (sodium chloride type structure) and silver structures occurs across the interface. The conclusion of Drabble is cited by Brú and Gharpurey [13, 14] in regard to their own experimental results.

In the deposition of silver on the $\{100\}$ face of rock salt at $450^{\circ} \mathrm{C}$ one sees again in the orientation not observed that sodium ions would be brought close to silver ions and the situation is the same as that just discussed (see fig. 5). It was pointed out in regard to the deposition of silver on the $\{110\}$ face of rock salt that if the silver were rotated through 90 degrees, the fit would be nearly perfect, but that this orientation is not found. The orientation of best fit which is not found experimentally would again involve a close approach of the silver ions to the sodium ions (see fig. 6).

As was also pointed out previously, Brück found that whereas the cubic face-centered metals grew with the $\{100\}$ plane resting on the $\{100\}$ of rock salt and oriented with their cube edges parallel to the rock-salt cube, the cubic body-centered metals grew on the contact surface turned at 45 degrees to the rock salt cube. From figure 4 it is clear that in the case of a cubic body-centered metal, there would be no interference from repulsion of sodium ions in depositing such a metal with its cube diagonal along the sodium chloride cube edge because there would be no deposit-metal ions in these positions.

We see that although Brück's rule points in the right direction, it needs to be extended. If we consider that electrostatic forces are involved, we must say that the arrangement is such as to give maximum bonding to the substrate for the largest aggregate that is capable of forming. One must then sum the attractive bonding energy, and the repulsive antibonding energy between each silver ion and its four nearest neighbor substrate ions of each species over the area that is defined by the points where exact fit is obtained between the substrate salt lattice and the deposit-metal salt lattice. If the sum of the attractive energies (-) added to the sum of the repulsive energies $(+)$ is negative, the arrangement is stable, but if it is positive, the arrangement in question should not exist. It should be possible to explain all of the orientations that have been found by determining these sums graphically both for these orientations and for other possible orientations which are not observed. It should also be possible to explain the laying down of the $\{100\}$ plane and the $\{110\}$ plane of the face-centered cubic metals parallel to the substrate at higher temperatures as higher energy configurations which are nevertheless stable (i. e., the sign of the sum is negative).

A small square region ( 15 cell lengths by 15 cell lengths) of silver-chloride lattice imposed on the rock-salt lattice has been mapped. A silver ion was placed directly over a chloride ion at the origin and actual measurement of $\mathrm{Ag}^{+}-\mathrm{Cl}^{-}$distances, and $\mathrm{Ag}^{+}-\mathrm{Na}^{+}$distances was carried out in the first quadrant only. At a cell having coordinates of seven 
cell lengths from the origin along both the ordinate and abscissa the misfit has become about 10 percent. The cell at this distance from the origin is shown in figure 9. Each silver ion in the area considered has four chloride ion neighbors and four sodium ion neighbors in the plane below the silver ion monolayer. Planar distances, which were measured, were converted to actual distances by means of a graph of planar distance versus actual distance for which the actual distances were computed by using a 20 -inch log-logvector slide rule. The bonding energy is the sum of $e^{2} / r$, where the distances, $r$, are those of the silver ions from their chloride ion neighbors. The antibonding energy is the sum of $e^{2} / r$, where the distances, $r$, are those of the silver ions from their sodium ion neighbors. In the counting process, each distance is counted twice except for the $\mathrm{Ag}^{+}-\mathrm{Cl}^{-}$distance at the center of the cube face. Where distances were counted twice, half the sum was taken. The result for the first row along the abscissa for seven and a half cells was $7.092 e^{2}$ for the bonding energy, and $4.821 e^{2}$ for the antibonding energy. The net bonding energy was $2.271 e^{2}$. For the seventh row above the abscissa where net the maximum misfit reached about 10 percent, the bonding energy had decreased only to $2.259 e^{2}$. At this rate of decrease a very large area could be covered before the antibonding energy became equal to the bonding energy. In this connection, Hass [39] has found that very thin silver films deposited on collodion exist as isolated granules $100 \mathrm{~A}$ to $150 \mathrm{~A}$ in diameter, but that thin silver films deposited on rock salt do not contract as strongly into isolated granules. Measurement of distances for only the first cell at the origin with the silver chloride turned at 45 degrees with respect to the rock salt cube gave an energy already slightly antibonding. Hence at a nucleus consisting of the first cell, this orientation became impossible.

To find if this basis for predicting epitaxial temperatures has any real value, Suddeth and McCraw have carried out evaporations of metals in a vacuum on rock salt at temperatures which their common ionization potential would indicate to be about correct on the graph of epitaxial temperature against ionization potential (see fig. 8). In the case of lead, which according to its ionization potential should have oriented at about $200^{\circ} \mathrm{C}$, a slight orientation was found at $225^{\circ} \mathrm{C}$. However, the results with lead were not reproducible even among separate deposits on the same cleavage surface which were made simultaneously and in no case was complete single crystal orientation obtained. It is possible that the misfit was too large and that better results would have been obtained on potassium chloride, but there was no potassium chloride single crystal on hand at the time to use for the purpose. Lead is cubic face-centered with $a=4.93$. Lead chloride is orthorhombic. The cell dimensions are: $a=9.030$, $b=7.608$, and $c=4.525$.

According to the graph of epitaxial temperature against ionization potential, antimony, with a common valence of three, should orient at about $380^{\circ} \mathrm{C}$.

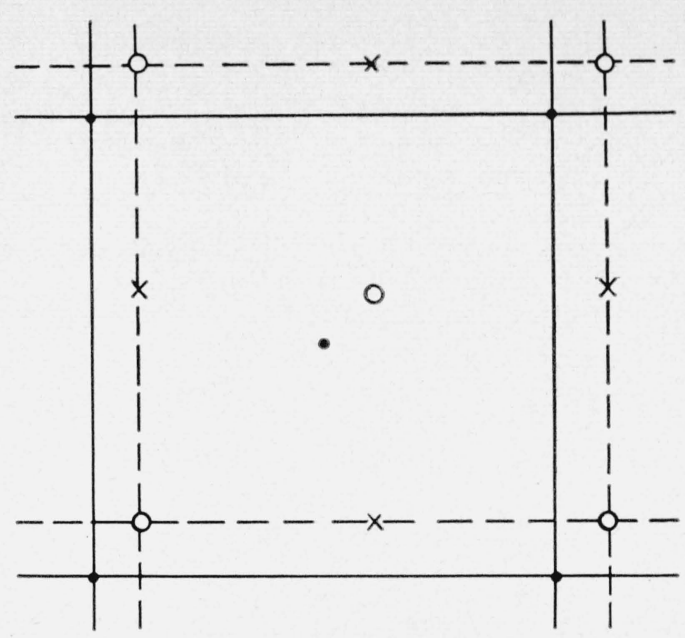

Figure 9. Misfitted silver ion lattice on rock salt. ๑, Silver ion; $\mathrm{X}$, sodium ion; $\bigcirc$, chloride ion

Suddeth and McCraw have found partial orientation for antimony from $250^{\circ}$ to $410^{\circ} \mathrm{C}$. They found appearances in the diffraction patterns similar to those observed by Brück for aluminum and gold, which he interpreted as the transition from deposition with the $\{111\}$ face parallel to the substrate to deposition with the $\{100\}$ face parallel to the substrate. Suddeth and McCraw have not as yet obtained a perfect single crystal of antimony having only the $\{100\}$ face parallel to the rock salt. Antimony has the arsenic-type lattice. For the antimony cell $a=4.50$, and the nearest neighbor distance $d$ is 2.87 . Data for antimony trichloride do not seem to be available. For $\mathrm{SbI}_{3}$ in a bimolecular cell, $a_{0}=8.186$ with $\alpha=54^{\circ} 14^{\prime}$ and in a hexagonal pseudo cell $a_{0}^{\prime}=7.466$ and $c_{0}^{\prime}=20.89$.

According to the graph of epitaxial temperature against ionization potential, platinum for an ionization state of two should orient at about $250^{\circ} \mathrm{C}$. Thirsk [40], by employing evaporation from a hot platinum wire, has obtained orientation of $\mathrm{Pt}$ on potassium chloride at the epitaxial temperature of $250^{\circ} \mathrm{C}$ with the $\mathrm{Pt}$-cube edge parallel to the cube edge of the substrate. The $\{100\}$ face of potassium chloride was used. The epitaxial temperature on rock salt will not be very different judging from the data of Brück where he used potassium chloride as substrate as well as sodium chloride. The data for the three metals for which he found the epitaxial temperature on both substrates are given in table 4 .

TABLE 4

\begin{tabular}{|c|c|c|}
\hline Substrate & $\begin{array}{l}\text { Metal } \\
\text { deposit }\end{array}$ & $\begin{array}{l}\text { Epitaxial } \\
\text { temperature }\end{array}$ \\
\hline $\begin{array}{l}\mathrm{NaCl}_{.} . . \\
\mathrm{KCl} . .\end{array}$ & $\begin{array}{l}\mathrm{Ag} \\
\mathrm{Ag}\end{array}$ & $\begin{array}{l}{ }^{\circ} \mathrm{C} \\
150 \\
150\end{array}$ \\
\hline $\begin{array}{l}\mathrm{NaCl}_{\mathrm{K}} \\
\mathrm{KCl}\end{array}$ & $\begin{array}{l}\mathrm{Pd} \\
\mathrm{Pd}\end{array}$ & $\begin{array}{l}250 \\
250\end{array}$ \\
\hline $\begin{array}{l}\mathrm{NaCl}_{\mathrm{KCl}} \\
\mathrm{KCl}\end{array}$ & $\begin{array}{l}\mathrm{Ni} \\
\mathrm{Ni}\end{array}$ & $\begin{array}{l}370 \\
410\end{array}$ \\
\hline
\end{tabular}


Brück pointed out in regard to these data that the temperature varied in the case of nickel where the misfit was large.

Consequently, it seems fairly certain that when an adequate method is used for depositing platinum on rock salt, it will be found to orient at a temperature close to that predicted by the graph of epitaxial temperature against ionization potential.

Suddeth and McCraw made an evaporation of tin at the epitaxial temperature which the graph would predict for the valence state of two. The result was negative with pure tin but showed considerable orientation in an impure sample which probably contained lead.

\section{Summary}

From an analysis of experimental data on the formation of oriented deposits of metals from their vapors in a vacuum onto salt substrates, it appears reasonable to suppose that a surface ionization process may explain the existence of metal ions that are brought into preferred orientations by attractive electrostatic forces that act between them and the subtrate ions. A relation between ionization potential and the epitaxial temperature at which the $\{100\}$ plane deposits parallel to the $\{100\}$ plane of the rock-salt substrate has been found which may be useful as a basis for making predictions as to the temperature at which orientation will occur. The results appear to indicate that the sequence of layers - substrate salt, deposit-metal salt monolayer, deposit-metal - exists.

In making a prediction of a substrate-metal pair and of the temperature at which orientation of the $\{100\}$ plane of the deposit parallel to the $\{100\}$ plane of the substrate should occur: (a) It is necessary to consider the lattice fit between the substrate salt and the deposit-metal salt, and the lattice fit between the metal salt and the metal. (Structure data for many of the metal salts in the anhydrous state are at present unavailable.) (b) The epitaxial temperature can be roughly estimated from the accompanying graph by using the most common ionization potential of the metal in question. But if the metal salt corresponding to this ionization potential is unstable, or if this salt cannot orient on the substrate salt, a higher ionization state (and correspondingly higher epitaxial temperature) may be required.

I express my appreciation to L. Marton for his continued interest in this work, and to Robert G. Breckenridge for his suggestions and encouragement.

\section{References}

[1] L. Holland, The production, properties, and uses of thin films condensed in vacuo, Vacuum 1, 23 (1951

[2] W. Lewis, Thin films and surfaces (Chemical Ptoblishing Co., New York, N. Y., 1950).

[3] L. Marton, Electron physics, NBS Circular 527 (in preparation); presented at American Physical Society (New York, N. Y., February 1952); Phys. Rev. 85, 1057 (1952).
[4] M. L. Royer, Recherches experimentales sur l'epitaxie ou orientation mutuelle de cristaux d'espèces differentes, Bul. soc. franç. minéral. 51, 7 (1928).

[5] G. W. Johnson, Surface tension effects in oriented thin silver films, J. Appl. Phys. 21, 449 (1950).

[6] C. Allen Sloat and Alan W. C. Menzies, Phenomena due to forces at crystal faces as studied by mutual orientation, J. Phys. Chem. [3] 35, 2004 (1931).

[7] F. C. Frank and J. H. van der Merwe, One-dimensional dislocations Part I. Static theory, Proc. Roy. Soc. [A] 198, 205 (1949).

[8] J. H. van der Merwe, Misfitting monolayers and oriented overgrowth, Discussions Faraday Soc. No. 5, 201 (1949).

[9] L. G. Schulz, Growth of alkali halide crystals from the vapor phase and from solution onto substrates of mica, Acta Cryst. 4, 483 (1951).

[10] D. W. Pashley, Oriented deposits on crystalline substrates, Proc. Phys. Soc. [A] 65, 33 (1952).

[11] L. Brück, Die Struktur dünner auf Steinsalz aufgedampfter Metallschichten, Ann. Physik 418, 233 (1936).

[12] M. K. Gharpurey, Doctoral Thesis, University of London, (1950). See references [13] and [14].

[13] L. Brú and M. K. Gharpurey, Expitaxial erystal growth of silver on rock salt (110) and (111) faces, Proc. Phys. Soc. [A] 64, 283 (1951).

[14] L. Brú and K. G. Gharpurey, Epitaxia de la plata sobre las caras (110) y (111) de la sal gema, Anales Real Soc. Espan. Fis. y Quim. [A] 4\%, 101 (1951).

[15] Ryozi Uyeda, Cathode-ray investigation of thin layers formed on some single crystals Part III. Silver films on sulphide crystals, Proc. Phys. Math. Soc. Japan 22, 1023 (1940); Part IV. Growth of submicroscopic silver crystals on rock salt, zincblende, and molybdenite, Proc. Phys. Math. Soc. Japan 24, 809 (1942).

[16] M. Smollett and M. Blackman, Lattice stability and oriented overgrowth, Proc. Phys. Soc. [A] 64, 683 (1951).

[17] D. S. Eppelsheimer and R. R. Penman, Thermal dilation of copper, Physica 16, 792 (1950).

[18] A. Neuhaus, Ưber orientierte Kristallabscheidung auf angeregten Tragerflächen, Naturwissenschaften $\mathbf{1 6}$, $31(1950)$.

[19] G. W. Johnson, Some observations on the epitaxy of sodium chloride on silver, J. Appl. Phys. 21, 1057 (1950)

[20] K. R. Dixit, Investigation of the orientation in thin evaporated metallic films by the method of electron diffraction, Phil. Mag. 16, 1049 (1933).

[21] G. P. Thomson and W. Cochrane, Theory and practice of electron diffraction (MacMillan and Co., Ltd., London, 1939).

[22] G. P. Thomson, The growth of crystals, Proc. Phys. Soc. 61, 403 (1948)

[23] H. Wilman, The slip, twinning, cohesion, growth, and boundaries of crystals, Proc. Phys. Soc. [A] 64, 329 (1951).

[24] Yoshiro Kainuma, Stucture of thin metallic films formed by evaporation on molybdenite, J. Phys. Soc. Japan 6, 135 (1951).

[25] Mieko Kubo and Shizuo Miyake, Electron-diffraction study on behavior of metals deposited on crystal surface. Part III. Effect of centers of crystallization on crystal growth in metal films, J. Phys. Soc. Japan 3, $114(1948)$.

[26] J. E. Lennard-Jones and Beryl M. Dent, Cohesion at a crystal surface, Trans. Faraday Soc. 24, 92 (1938).

[27] T. N. Rhodin, Jr., Oriented arrangements of thin aluminum films formed on ionic substrates, Discussions Faraday Soc., No. 5, 215 (1949).

[28] T. N. Rhodin, Jr., Oriented arrangements of thin aluminum films on ionic substrates, Trans. Am. Inst. Mining Met. Engrs. 185, 371 (1949).

[28a] Olive G. Engel, A model for epitaxy in metal-rock salt pairs, J. Chem. Phys. 20, 1174 (1952).

[29] Clarence Zener, Theory of diffusion in "Imperfections in nearly perfect crystals'" (John Wiley \& Sons, Inc. New York, N. Y., 1952). 
[30] N. F. Mott and R. W. Gurney, Electronic processes in ionic crystals, 2d ed., p. 111 (Oxford University Press, Oxford, England, 1948).

[31] K. F. Herzfeld, Eine elektrostatische Überschlagrechnung zur obigen Arbeit, Z. physik. Chem. 105, 329 (1923).

[32] Atomic Energy Levels, NBS Circular 467, I and II.

[33] D. A. Wright, Work function and energy levels in insulators, Proc. Phys. Soc. 60, 13 (1948).

[34] M. A. Catalan and F. R. Rico, Anales Real Soc. Espan. de Fis. y Quim. [A] 68, 328 (1952).

[35] G. W. Johnson, The epitaxy of alkali chlorides on metals, J. Appl. Phys. 22, 797 (1950).

[36] H. Wilman, The structure and orientation of silver halides, Proc. Phys. Soc. 52, 323 (1940).
[37] Harold H. Morris, A study of the chemistry of gold at high temperatures and pressures, J. Am. Chem. Soc. 40, 917 (1918).

[38] J. R. Drabble, Doctoral Thesis, University of London (1949). See references [13] and [14].

[39] G. Hass, Untersuchungen über den Aufbau aufgedampfter Metallschichten mittels Ưbermikroskop und Elektroneninterferenzen, Kolloid-Z. 100, 230 (1942).

[40] H. R. Thirsk, A note on the orientated overgrowth of metal films on single crystal inorganic substrates, Proc. Phys. Soc. 63, 833 (1950).

Washington, March 4, 1953. 